\title{
Kein Fortschritt ohne randomisierte, kontrollierte Studien
}

Fragestellung: Wie wirksam ist eine Kombinationschemotherapie aus Temozolomid und Methotrexat kombiniert mit Rituximab gefolgt von Ganzhirnbestrahlung in der Primärtherapie primärer ZNS-Lymphome?

Hintergrund: Bis auf den Einsatz von hochdosiertem Methotrexat sind alle Therapiemaßnahmen in der Primärtherapie der ZNS-Lymphome umstritten und nicht gesichert. Aktuelle Bemühungen konzentrieren sich vor allem auf eine Intensivierung der medikamentösen Therapie durch Alkylanzien oder andere Zytostatika sowie den Einsatz des B-Zell-depletierenden Antikörpers Rituximab. Die Strahlentherapie ist nach den Ergebnissen der G-PCNSL-SG-1-Studie in der Primärtherapie dieser Tumoren obsolet.

Patienten und Methoden: Im Phase-I-Teil der Studie wurde die tolerierte Dosis von Temozolomid im Rahmen der Kombinationstherapie bestimmt. Im Phase-II-Teil der Studie erhielten die Patienten Temozolomid $\left(100 \mathrm{mg} / \mathrm{m}^{2}\right)$, Methotrexat $(3,5 \mathrm{~g} /$ $\left.\mathrm{m}^{2}\right)$ und Rituximab $\left(375 \mathrm{mg} / \mathrm{m}^{2}\right)$ in Zyklen von vier Wochen.

Die Ganzhirnbestrahlung mit 36 Gy (zweimal 1,2 Gy

Glass J, Won M, Schultz CJ et al. Phase I and II study of induction chemotherapy with methotrexate, rituximab and temozolomide, followed by whole-brain radiotherapy and postirradiation temozolomide for primary CNS lymphoma: NRG Oncology RTOG 0227. J Clin Oncol 2016; 34: $1620-5$ täglich), begann in Woche 11, Temozolomid wurde danach fortgeführt.

Ergebnisse: Insgesamt $13 \mathrm{~Pa}$ tienten durchliefen die Phase I, die maximal tolerierte Dosis von Temozolomid betrug $100 \mathrm{mg} / \mathrm{m}^{2}$. Dosislimitierende Toxizitäten waren hepa-

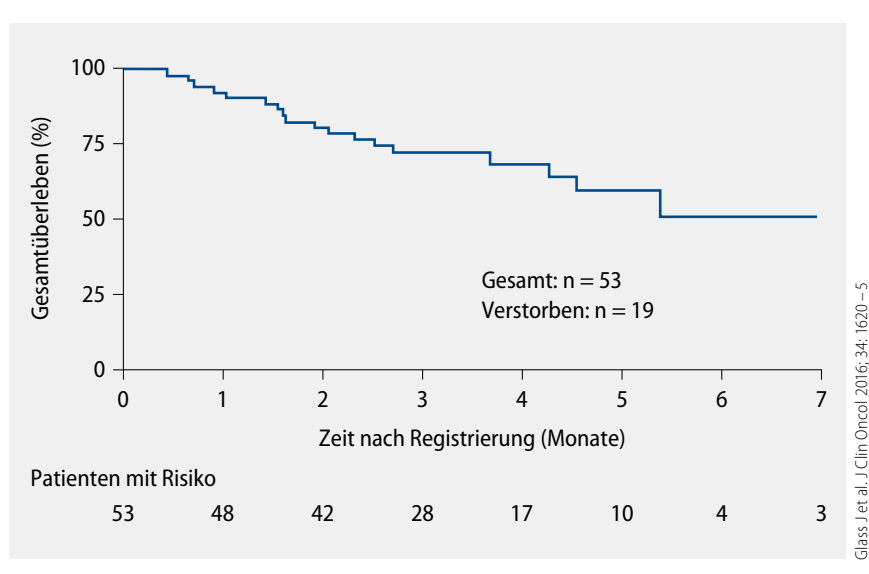

1 Gesamtüberleben im Phase-II-Studienteil: 19 von 53 Patienten verstarben im medianen Nachbeobachtungszeitraum von 3,6 Jahren.

tisch und renal. Im Phase-II-Studienteil wurden 53 Patienten behandelt. Bei einer medianen Nachbeobachtungszeit von 3,6 Jahren betrugen das progressionsfreie und Gesamtüberleben nach zwei Jahren $63,6 \%$ beziehungsweise $80,8 \%$ ( $\triangleright$ Abb. 1). Die objektive Ansprechrate betrug 85,7\% im Phase-II-Teil. Kognitive Funktionen und Lebensqualität verbesserten oder stabilisierten sich nach der Ganzhirnbestrahlung.

Schlussfolgerungen: Die Autoren meinen, dass diese Therapie sicher sei und dass sie das beste Ergebnis für progressionsfreies und Gesamtüberleben von allen RTOG-Studien erzielt hätte. Sie räumen ein, dass randomisierte Studien notwendig sind, um die Wirksamkeit dieser Therapiestrategie zu bestätigen.

\section{- Kommentar von Michael Weller, Zürich, Schweiz}

\section{Kein neuer Therapiestandard}

Es handelt sich um eine weitere einarmige Studie zur Behandlung der primären ZNS-Lymphome, die das Feld nicht weiterbringen wird. Der Einsatz der Strahlentherapie in der Primärtherapie ist obsolet, aber man muss einräumen, dass diese RTOG-Studie vor den Ergebnissen der Deutschen G-PCNSLSG1-Studie begonnen wurde. Dennoch bleibt es bedauerlich, dass selbst große Studiengruppen wie die RTOG weiterhin einarmige Studien bei einer Erkrankung durchführen und publizieren, bei der sich seit langem und immer wieder zeigt, dass ohne Randomisierung kein Fortschritt zu erzielen ist. Das vorliegende Regime mit Ganzhirnbestrahlung als potenziellen neuen Therapiestandard vorzuschlagen (letzter Absatz der Diskussion) ist deshalb nicht nachvollziehbar.

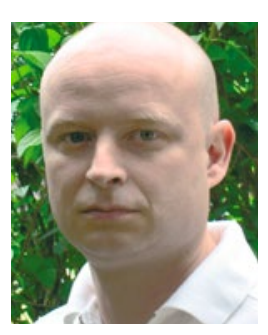

Prof. Dr. med. Michael Weller, Zürich/Schweiz

Direktor der Klinik für Neurologie, Universitätsspital Zürich E-Mail: michael.weller@usz.ch 